\title{
Awareness on Platelet Rich Plasma Among Dental Students - A Survey
}

\author{
Hemashree J', Dhanraj Ganapathy² and Revathi Duraisamy ${ }^{3}$ \\ ${ }^{1}$ Department of Prosthodontics, Saveetha Dental College and Hospitals, Saveetha Institute \\ of Medical and technical sciences(SIMATS), Saveetha University,Chennai, India. \\ ${ }^{2}$ Professor and Head, Department of Prosthodontics, Saveetha Dental College and Hospitals, Saveetha \\ Institute of Medical and technical sciences (SIMATS), Saveetha university, Chennai, India. \\ ${ }^{3}$ Senior lecturer, Department of Prosthodontics, Saveetha Dental College and Hospitals, Saveetha \\ Institute of Medical and technical sciences (SIMATS) Saveetha University, Chennai, India
}

\section{ABSTRACT}

Platelet rich plasma (PRP), also called as autologous platelet gel, is nothing but plasma rich in growth factors , platelet concentrate. In other words, it is an increased concentration of platelets suspended in a small amount of plasma after the process of centrifugation. This survey aimed at assessing the knowledge and awareness of platelet rich plasma among dental students. The study was done in an online setting among the dental students of the Chennai population. The sample size of 150 participants of age group 20-23 years, both males and females were selected by a simple random sampling method. Both descriptive ( frequency of the responses) and inferential statistics (Chi - square tests) were done and the results were presented in the forms of graphs. According to this survey based study it was noted that the females (64\%) have participated in higher numbers compared to males (36\%). It was noted that 59.33\% of the population participated in the study were aware of the term PRP (ie. platelet rich plasma) whereas $40.67 \%$ of the population was not aware of the term. The association of gender and awareness of platelet rich plasma among the participants was found to be statistically significant, $p=0.000(<0.05)$. Within the limitations of this study, it was inferred that the knowledge and awareness of platelet rich plasma was different among both the genders. More appropriately, females were much aware compared to males. Association of gender and awareness of platelet rich plasma among the participants was found to be statistically significant.

KEY WORDS: AWARENESS; PLATELET RICH PLASMA; GENDER; GROWTH FACTORS.

\section{INTRODUCTION}

Platelet rich plasma (PRP), also called as autologous platelet gel, is nothing but plasma rich in growth factors, platelet concentrate. In other words, it is an increased

\section{ARTICLE INFORMATION}

*Corresponding Author: dhanraj@saveetha.com

Received 25th June 2020 Accepted after revision 10th August 2020 Print ISSN: 0974-6455 Online ISSN: 2321-4007 CODEN: BBRCBA

Thomson Reuters ISI Web of Science Clarivate Analytics USA and Crossref Indexed Journal

\section{Clarivate
Analytics}

NAAS Journal Score 2020 (4.31) SJIF: 2020 (7.728)

A Society of Science and Nature Publication,

Bhopal India 2020. All rights reserved.

Online Contents Available at: http//www.bbrc.in/

Doi: http://dx.doi.org/10.21786/bbrc/13.7/7 concentration of platelets suspended in a small amount of plasma after the process of centrifugation. Basically, a patient's own blood is collected and is made to undergo the process of centrifuging at varying speed until it separates into 3 different layers i.e platelet poor plasma, platelet rich plasma, and red blood cells. Usually 2 spins are required. The first spin also known as the hard spin separates the platelet poor plasma from the red fraction and platelet rich plasma. The second spin also known as the soft spin separates the red fraction from the platelet rich plasma. The material with the highest specific gravity (platelet rich plasma) will be deposited at the bottom of the tube. Immediately prior to application of this process , a platelet agonist such as the 10\% calcium chloride and 
topical bovine thrombin is added to activate the clotting cascade, producing a platelet gel.

This whole process of preparation takes approximately 12 minutes and produces a platelet concentration of 3-5 times that of native plasma(Marx et al., 1998; Petrungaro, 2001). Platelets play a very fundamental role in hemostasis and are a natural source of growth factors. Growth factors, present within platelet $\alpha$-granules, comprise platelet derived growth factor (PDGF), insulin like growth factor (IGF), vascular endothelial growth factor (VEGF), platelet derived angiogenic factor (PDAF), and transforming growth factor beta (TGF- $\beta$ ) (Bennett and Schultz, 1993). The release of these growth factors is triggered by the activation of platelets that can be initiated by a variety of substances or stimuli such as thrombin, calcium chloride, or collagen. Growth factors are concerned in main stages of wound healing and regenerative processes including chemotaxis, proliferation, differentiation, and angiogenesis. Consistent with the definition of platelet rich plasma, it's going to be assumed that these growth factors are present at increased concentrations in PRP.

In addition to growth factors (GFs), platelets release numerous other substances (e.g., fibronectin, vitronectin, sphingosine 1-phosphate, etc.) that are vital in wound healing. An advantage of platelet rich plasma over the utilization of single recombinant human protein delivery is that the release of multiple growth factors and differentiation factors upon platelet activation (Sánchez et al., 2003). Recently, the morphologic and molecular configuration of PRP was reported, it showed PRP may be a fibrin framework over platelets that has the potential to support regenerative matrix(El-Sharkawy et al., 2007; Fernández-Barbero et al., 2006). The underlying principle for using PRP in soft and hard augmentation are to hasten vascularization of the graft, advance soft tissue healing, reduce postoperative morbidity, and enhance bone regeneration(Anitua, 1999). Advantages of using an autologous PRP include no risk of cross reactivity, immune response or disease transmission(Weibrich et al., 2001). Furthermore , the utilization of PRP improve behavior of particulate graft materials and easier stuffing into a grafting site, thus facilitating space maintenance and potential bone regeneration(Freymiller and Aghaloo, 2004; Jakse et al., 2003).

Platelet-rich plasma (PRP) is currently considered as one of the main strategies to promote musculoskeletal tissues/collagen repair. There are several reports in the review of literature evidencing its potential in clinical trials as well as in vitro analysis(Hammond et al., 2009; Kajikawa et al., 2008; Schallmoser et al., 2007; Weibrich et al., 2001) . It is deployed as a cost-effective source of autologous growth factors that might affect stem cells proliferation and differentiation, as it is being increasingly investigated as an adjuvant or scaffold for stem cells-based therapeutics. However, the lack of standardization for the methodology of obtaining and using platelet rich plasma among different groups may hamper the development of this trending technology.
Previously our department has published extensive research on various aspects of prosthetic dentistry (Anbu et al., 2019; Ariga et al., 2018; Ashok and Ganapathy, 2019; Duraisamy et al., 2019; Ganapathy et al., 2017; Gupta et al., 2018; Jain, 2017a, 2017b; Ranganathan et al., 2017; Varghese et al., 2019; World Journal of Dentistry, 2017), this vast research experience has inspired us to research on the awareness platelet rich plasma among health practitioners. The present study aimed at assessing the knowledge and awareness of platelet rich plasma among dental students.

\section{MATERIAL AND METHODS}

The study was done in an online setting among the dental students of the Chennai population during December 2019. Institutional review board approval was obtained for this survey based analysis. 2 reviewers [Primary investigator \&t guide] were involved in this study. The sample size of 150 participants of age group 20-23 years, both males and females were selected by a simple random sampling method. Students pursuing internships in various dental institutions were given access to an online link and the responses were collected through an online database. Randomisation [for all variables] was followed to minimise the bias. Pretested questionnaires where the internal validity was the homogenisation and replication of experiment. Cross verification with existing studies was the external validity of this study. The results were collected and tabulated. Then the results were exported for statistical analysis to SPSS statistical software. Both descriptive ( frequency of the responses) and inferential statistics (Chi - square tests) were done and the results were presented in the forms of pie charts and bar graphs.

\section{RESULTS AND DISCUSSION}

According to this survey based study it was noted that the females(64\%) have participated in higher numbers compared to males(36\%) [Figure 1]. It was noted that $59.33 \%$ of the population participated in the study were aware of the term PRP (ie. platelet rich plasma) whereas $40.67 \%$ of the population was not aware of the term[ Figure2]. When the students were asked about application of platelet rich plasma in dentistry $36.67 \%$ of the population opted for endodontic followed by $28 \%$ for the field of surgery , 21.33\% for the field of implants and 14\% for the field of periodontics[Figure3]. 36\% of the population participated in the study were not aware of the components of platelet rich plasma[Figure 4]. Regarding the method of preparation of platelet rich plasma, only about $43.33 \%$ of the population were aware about the proper protocol for preparation of platelet rich plasma and $28.67 \%$ of the population had no idea about the preparation of platelet rich plasma[Figure 5]. About 36\% of the population were aware that platelet rich plasma is completely safe and does not face any host rejection[Figure 6]. 
Figure 1: Pie chart showing percentage distribution of the population according to gender participated in the study. $64 \%$ were females (Blue) whereas only 36\% males(Green) participated in the study. $\mathrm{N}=150$. Females have participated in higher numbers in this survey.

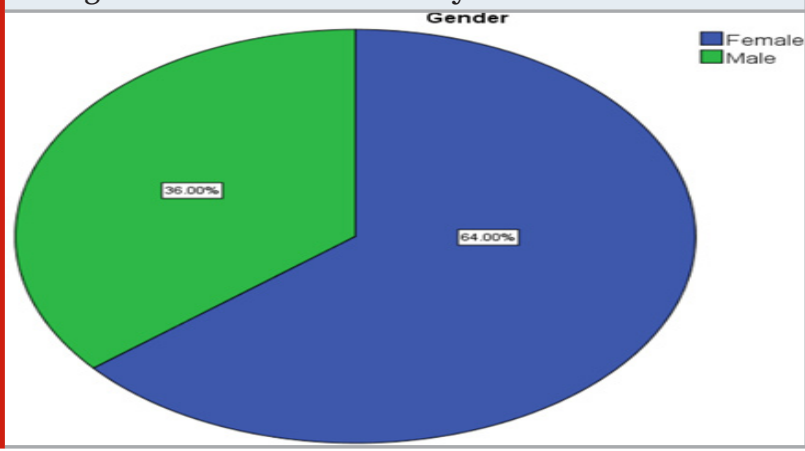

Figure 2: Pie chart showing percentage distribution of the awareness of the term Platelet rich plasma among the participants participated in the study. 59.33\% were aware of the term (Blue) whereas $40.67 \%$ were not aware of the term(Green). $N=150$. There is increased awareness of Platelet rich plasma among the study participants.

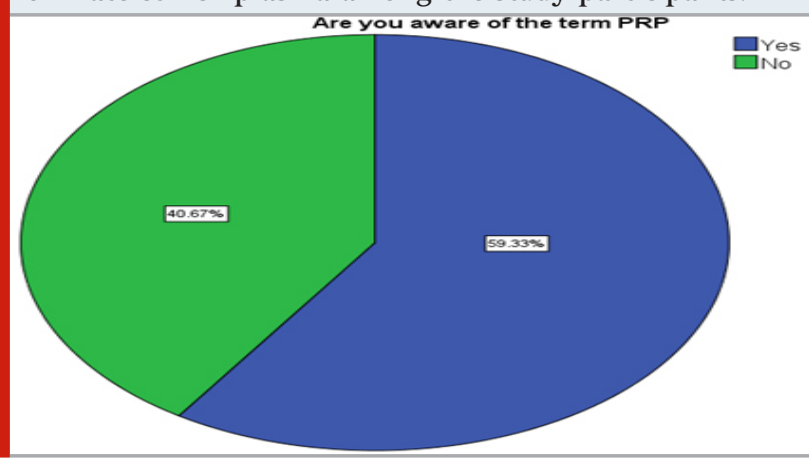

Figure 3: Pie chart showing percentage distribution of the awareness of the application of Platelet rich plasma in different fields of dentistry. 36.67\%(Purple) of the population opted for endodontics followed by $28 \%$ (Green) for the field of surgery, $21.33 \%$ (Beige) for the field of implants and 14\%(Blue) for the field of periodontics. $\mathrm{N}=150$. There was no clear idea of the application of Platelet rich plasma among the study participants.

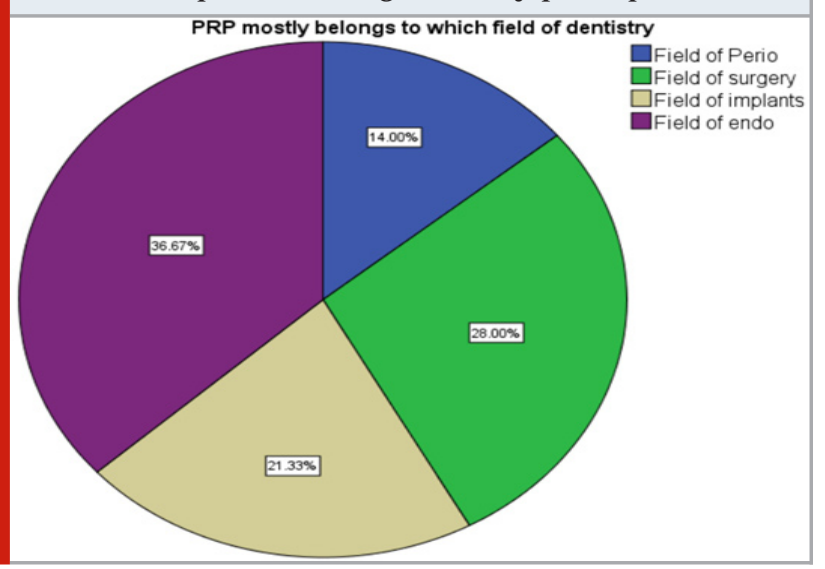

Figure 4: Pie chart showing percentage distribution of the awareness of the components of Platelet rich plasma among the participants participated in the study. 64\% were aware of the term (Blue) whereas 36\% were not aware of the components(Green). $N=150$. There is increased awareness of the components of Platelet rich plasma among the study participants.

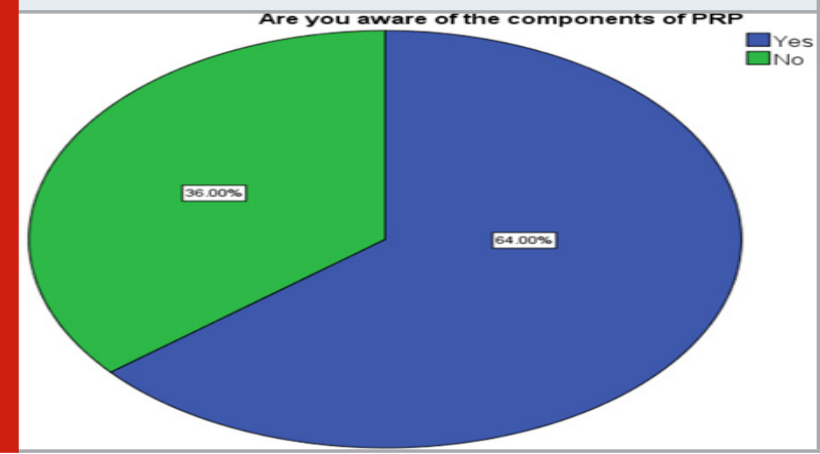

Figure 5: Pie chart showing percentage distribution of the awareness of the method of preparation of Platelet rich plasma. 43.33\%(Blue) of the population were aware about the proper protocol for preparation of platelet rich plasma and $28.67 \%$ (Purple) of the population had no idea about the preparation of platelet rich plasma. $N=150$. There is awareness of the method of preparation of Platelet rich plasma among the study participants.

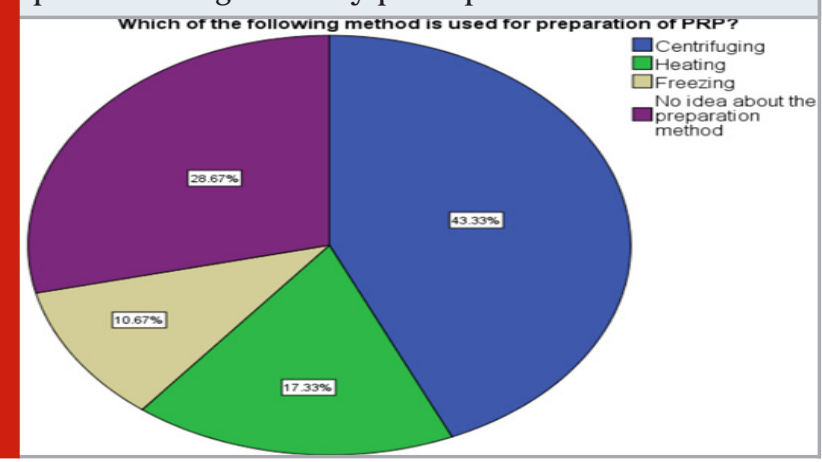

Figure 6: Pie chart showing percentage distribution of the awareness of Platelet rich plasma regarding host rejection among the participants participated in the study. 64\% were not aware (Green) whereas 36\% were aware of it (Blue). $\mathrm{N}=150$. There is not much knowledge regarding the compatibility of Platelet rich plasma among the study participants.

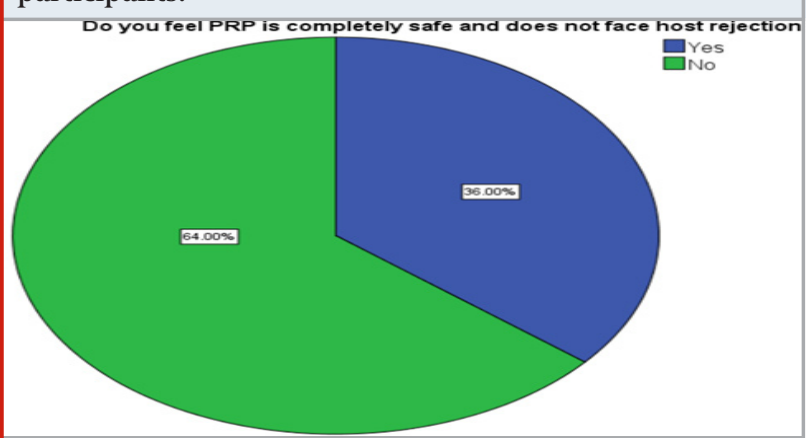


About 56\% of the participants included in the study were aware that platelet rich plasma can be used to treat BRONJ( Bisphosphonates related radionecrosis of the jaw)[ Figure 7]. 52\% of the population did not know that PRP injections are used to treat certain joint diseases like osteoarthritis [Figure 8]. Students were aware about the term fibrin sealants(67.33\%)[Figure 9]. 53.33\% of the patients knew that PRP is superior to fibrin sealants in their characteristics[Figure 10]. Association of gender and awareness of platelet rich plasma was found to be statistically significant with a p value of 0.000 [Figure11]. Association between gender and the field of dentistry in which platelet rich plasma is mostly applied was found to be statistically significant $(\mathrm{p}=0.007)$ [Figure12]. Association of gender and awareness of fibrin sealants was found to be statistically significant with a $\mathrm{p}$ value of 0.000[Figure13].within the study were aware of the term PRP (ie. platelet rich plasma) whereas $40.67 \%$ of the population wasn't aware of the term[ Figure2].

Figure 7: Pie chart showing percentage distribution of the awareness of Platelet rich plasma in BRONJ (Bisphosphonates related radionecrosis of the jaw) treatment among the participants participated in the study. 56\% were aware (Blue) whereas 44\% were not aware of it(Green). $\mathrm{N}=150$. There is knowledge regarding the use of Platelet rich plasma for BRONJ among the study participants.

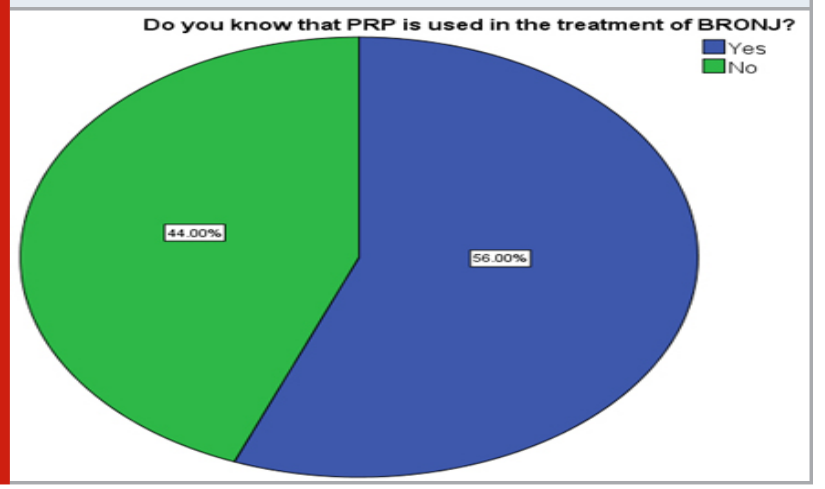

Figure 8: Pie chart showing percentage distribution of the awareness of Platelet rich plasma in treatment of joint disease among the participants participated in the study. $48 \%$ were aware (Blue) whereas 52\% were not aware of it(Green). $\mathrm{N}=150$. There is less knowledge regarding the use of Platelet rich plasma for treatment of joint diseases among the study participants.

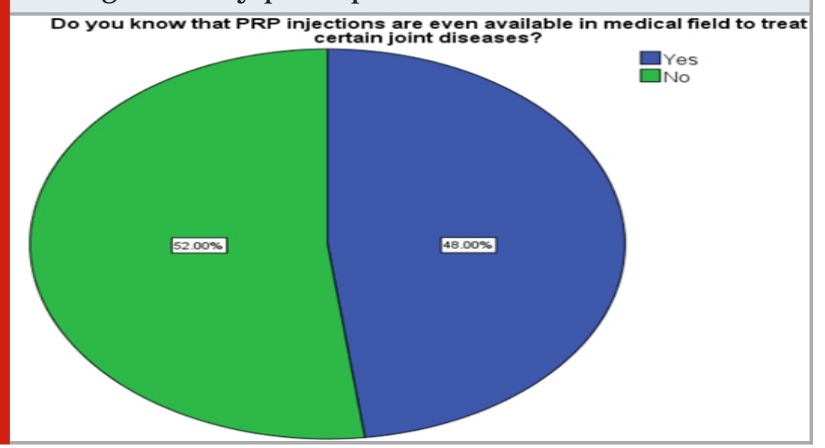

Figure 9: Pie chart showing percentage distribution of the awareness of fibrin sealants among the participants participated in the study. 67.33\% were aware (Blue) whereas $32.67 \%$ were not aware of it(Green). $\mathrm{N}=150$. There is knowledge regarding fibrin sealants among the study participants.

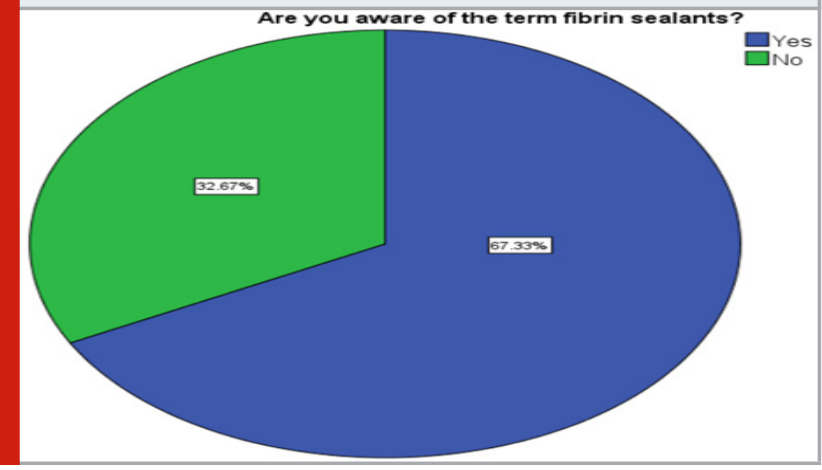

Figure 10: Pie chart showing percentage distribution of the awareness of properties of fibrin sealants versus platelet rich plasma among the participants participated in the study. $53.33 \%$ were aware that platelet rich plasma is superior to fibrin sealants(Blue) whereas $46.67 \%$ were not aware of it(Green). $\mathrm{N}=150$. There is knowledge regarding fibrin sealants among the study participants.

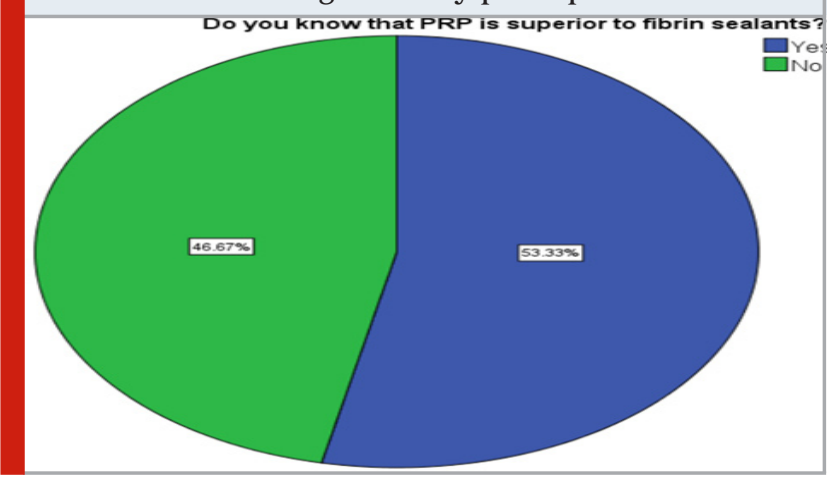

Figure 11: The bar graph represents the association of gender and awareness of platelet rich plasma among the participants. The $\mathrm{X}$ - axis represents the gender and the $\mathrm{Y}$ - axis represents the number of participants with their responses. Among the total participants, $46 \%$ of the females were aware of platelet rich plasma. This association was statistically significant.(Pearson Chi square value: $17.384, p=0.000(<0.05)$. Hence it is statistically significant.

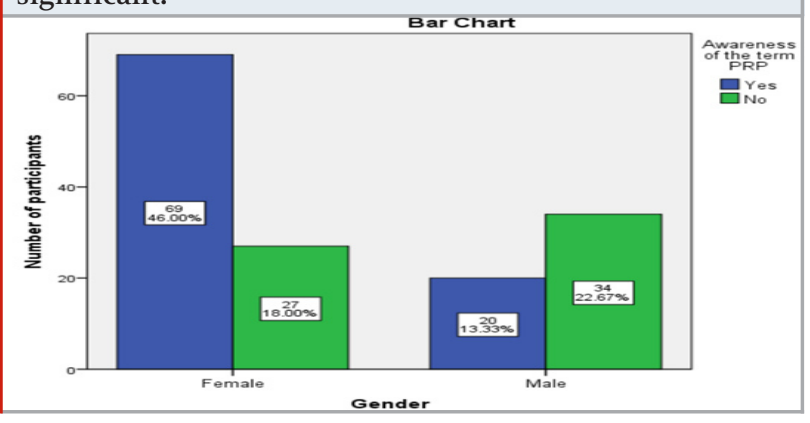


When the students were asked about application of platelet rich plasma in dentistry $36.67 \%$ of the population opted for endodontic followed by $28 \%$ for the world of surgery , 21.33\% for the world of implants and 14\% for the world of periodontics[Figure3]. 36\% of the population participated within the study weren't aware of the components of platelet rich plasma[Figure 4]. Regarding the tactic of preparation of platelet rich plasma, only about $43.33 \%$ of the population were conscious of the proper protocol for preparation of platelet rich plasma and $28.67 \%$ of the population had no idea about the preparation of platelet rich plasma[Figure 5]. About 36\% of the population were aware that platelet rich plasma is completely safe and doesn't face any host rejection[Figure 6]. About 56\% of the participants included within the study were aware that platelet rich plasma are often used to treat BRONJ(Bisphosphonates related radionecrosis of the jaw)[ Figure 7]. 52\% of the population didn't know that PRP injections are used to treat certain joint diseases like osteoarthritis [Figure 8].

Figure 12: The bar graph represents the association of gender and awareness of the field of application of platelet rich plasma in dentistry among the participants. The $X$ axis represents the gender and the $Y$ - axis represents the number of participants with their responses. Among the total participants, $30 \%$ of the females opted that platelet rich plasma was used in endodontics. This association was statistically significant.(Pearson Chi square value:12.111, $\mathrm{p}=0.007(<0.05)$. Hence it is statistically significant.

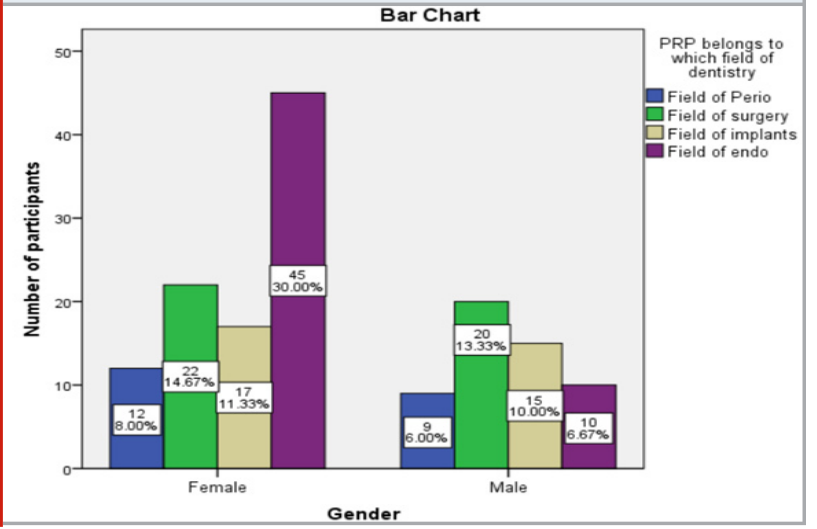

Students were conscious of the term fibrin sealants(67.33\%) [Figure 9]. 53.33\% of the patients knew that PRP is superior to fibrin sealants in their characteristics[Figure 10]. Association of gender and awareness of platelet rich plasma was found to be statistically significant with a $p$ value of 0.000 [Figure11]. Association between gender and thus the sector of dentistry during which platelet rich plasma is typically applied was found to be statistically significant( $p=0.007$ )[Figure12]. Association of gender and awareness of fibrin sealants was found to be statistically significant with a $p$ value of 0.000 [Figure13].

According to this survey, it was inferred that the knowledge of platelet rich plasma was different among both the genders. More appropriately, females were much aware compared to males.
Figure 13: The bar graph represents the association of gender and awareness of application of fibrin sealants among the participants. The $\mathrm{X}$ - axis represents the gender and the $Y$ - axis represents the number of participants with their responses. Among the total participants, 64\% of the females were aware of fibrin sealants. This association was statistically significant. (Pearson Chi square value:129.373, $\mathrm{p}=0.000(<0.05)$. Hence it is statistically significant.

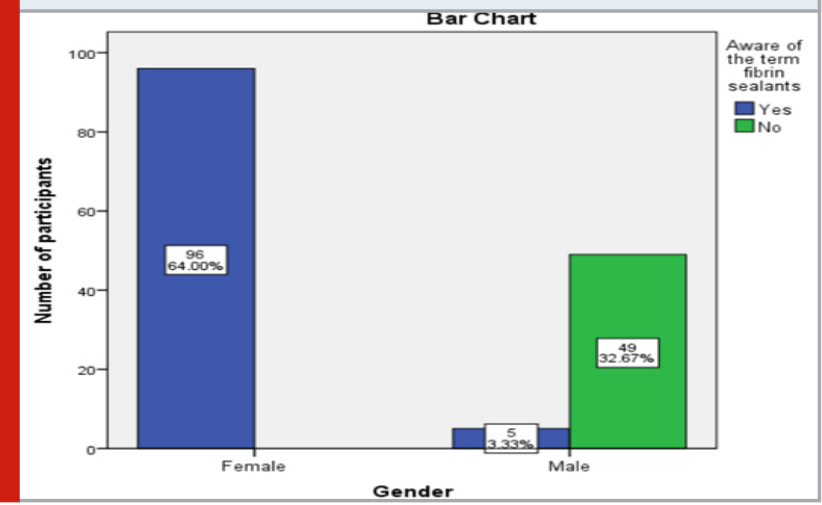

Figure 14: The bar graph represents the association of gender and awareness of properties of fibrin sealants versus platelet rich plasma among the participants. The $\mathrm{X}$ - axis represents the gender and the $\mathrm{Y}$ - axis represents the number of participants with their responses. Among the total participants, $53.3 \%$ of the females were aware that platelet rich plasma is superior to fibrin sealants. This association was statistically significant. (Pearson Chi square value:96.429, $\mathrm{p}=0.000(<0.05)$. Hence it is statistically significant.

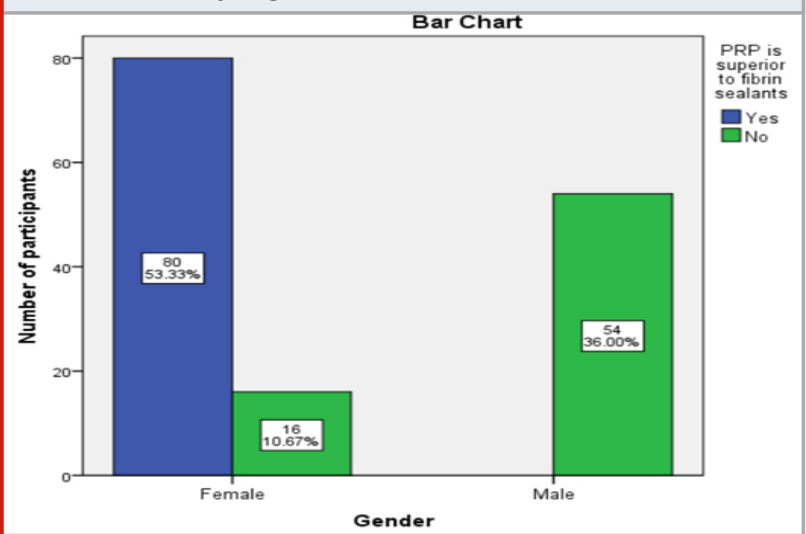

Platelet rich plasma can be used in different fields of dentistry and not specific to any particular field as such. The development of an autologous platelet rich plasma is proved to be relatively easy and ultimately effective as a surgical supplement, in order to retain desired levels of the growth factors after preparation. The potential use of Platelet rich plasma is to be clinically effective in acceleration of postsurgical healing in both periodontal and oral surgery applications mainly (Carlson and Roach, 2002). 
Figure 15: The bar graph represents the association of gender and awareness of the components of platelet rich plasma among the participants. The $\mathrm{X}$ - axis represents the gender and the $\mathrm{Y}$ - axis represents the number of participants. Among the total participants, 64\% of the females were aware of the components of platelet rich plasma. This association was statistically significant. (Pearson Chi square value: $150.000, \mathrm{p}=0.000(<0.05)$. Hence it is statistically significant.

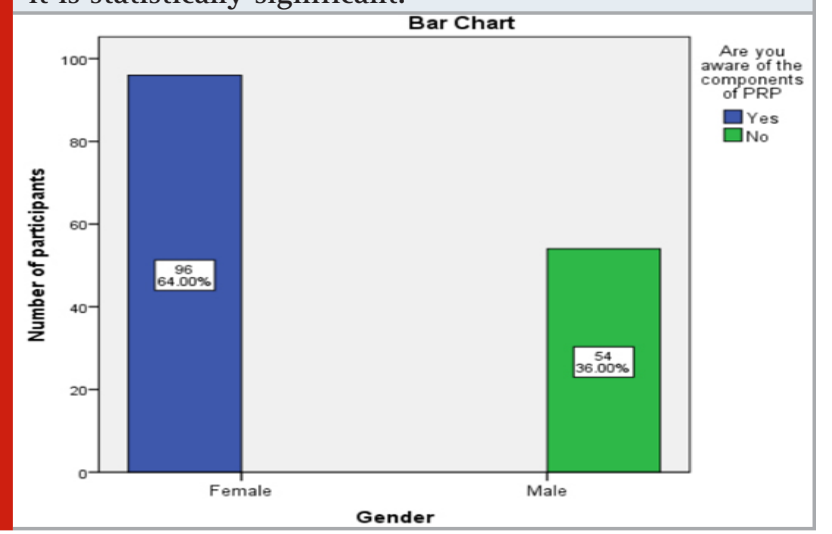

Components of platelet rich plasma are mainly the platelets, leucocytes, and growth factors. Several authors have tried to characterize and classify the numerous techniques available in the present use in terms of preparation (use of anticoagulant;centrifugation speed), components , and its applications (Anitua, 1999; Dohan Ehrenfest et al., 2009). Regarding the techniques of preparation most of the responses said it was centrifuging. Though it is correct the method of preparation is explained briefly as follows: Open technique: the product is exposed during preparation to the environment and comes in direct contact with different materials that are to be used for their production, like the pipettes or any kind of product-collecting tubes. It is specifically ensured that with the open technique, it is not to be contaminated during any kind of microbiological handling. Closed technique: it involves the use of commercial available devices with markings in which the preparation is not exposed to the environment . closed technique is the most safe and preferred method of preparation of platelet rich plasma.

Platelet rich plasma has a wide range of applications other than dental field. It is used in the dermatological field for the purpose of alopecia treatment, in BRONJ, in Osteoarthritis and so on. A study done by Mauceri et al (Mauceri et al., 2018), showed that platelet rich plasma as an adjunct to laser can cause effective healing. In this study in order to manage BRONJ patients performing the conservative type of treatment through Er,Cr:YSGG laser combined platelet rich plasma,will enhance the bone and mucosal healing, with a successful outcome of $80 \%$.The use of laser technology for BRONJ treatment and its advantages on tissue healing capacity has been widely investigated in the past years (Ghidini et al., 2017; Latifyan et al., 2016). It can improve post surgical wound healing by stimulating the release of growth factors ;promoting angiogenesis and bone healing. It is also implicated in the treatment of osteoarthritis . platelet rich plasma in addition to hyaluronic acid, dehydrated human amniotic/chorionic membrane tissue, have started to gain traction for osteoarthritis. Since platelet rich plasma is considered to have both anti-inflammatory effects through growth factors such as transforming growth factor- $\beta$ and insulin-like growth factor 1 , and stimulatory effects on mesenchymal stem cells and fibroblasts they can play a pivotal role(Southworth et al., 2019) .

Fibrin sealant back in the 90's was the first modern era material approved as a hemostat in the. It was the only agent approved as a hemostat, sealant, and adhesive by the Food and Drug Administration (FDA)(Spotnitz, 2014). The product is presently supplied in forms of patches additionally to the primary liquid formulations. However, with the passage of time newer products came into use. One such thing was platelet rich plasma. In an in vitro study in the dogs(Hermeto et al., 2012) showed that platelet rich plasma is clinically superior to the fibrin sealants, when these agents were used on full-thickness skin grafts. It has been emphasized that the histological analysis demonstrated greater presence of fibroblasts in the group treated with platelet-rich plasma.

PRP is now being widely used as a new therapeutic option in the field of dermatology, such as trichology, wound healing, and cosmetic medicine. In this manner, understanding the biological characteristics and mechanism of action of this platelet rich plasma will help clinicians in selecting a system that meets their specific needs for a given indication. Further clinical trials and studies need to be evaluated for the purpose of usage of platelet rich plasma.

\section{CONCLUSION}

Within the limitations of this study, it was inferred that the knowledge and awareness of platelet rich plasma was different among both the genders. More appropriately, females were much aware compared to males. Association of gender and awareness of platelet rich plasma among the participants was found to be statistically significant.

\section{Conflict of Interests: None declared.}

\section{REFERENCES}

Anbu RT, Suresh V, Gounder R, et al. (2019) Comparison of the Efficacy of Three Different Bone Regeneration Materials: An Animal Study. European journal of dentistry 13(1): 22-28.

Anitua E (1999) Plasma rich in growth factors: preliminary results of use in the preparation of future sites for implants. The International journal of oral $\mathrm{ct}$ maxillofacial implants 14(4): 529-535.

Ariga P, Nallaswamy D, Jain AR, et al. (2018) Determination of Correlation of Width of Maxillary Anterior Teeth using Extraoral and Intraoral Factors in 
Indian Population: A Systematic Review. World Journal of Dentistry 9(1): 68-75.

Ashok V and Ganapathy D (2019) A geometrical method to classify face forms. Journal of oral biology and craniofacial research 9(3): 232-235.

Bennett NT and Schultz GS (1993) Growth factors and wound healing: biochemical properties of growth factors and their receptors. American journal of surgery 165(6): 728-737.

Carlson NE and Roach RB Jr (2002) Platelet-rich plasma: clinical applications in dentistry. Journal of the American Dental Association 133(10): 1383-1386.

Dohan Ehrenfest DM, Rasmusson L and Albrektsson $\mathrm{T}$ (2009) Classification of platelet concentrates: from pure platelet-rich plasma (P-PRP) to leucocyte- and platelet-rich fibrin (L-PRF). Trends in biotechnology 27(3): 158-167.

Duraisamy R, Krishnan CS, Ramasubramanian H, et al. (2019) Compatibility of Nonoriginal Abutments With Implants: Evaluation of Microgap at the ImplantAbutment Interface, With Original and Nonoriginal Abutments. Implant dentistry 28(3): 289-295.

El-Sharkawy H, Kantarci A, Deady J, et al. (2007) Platelet-rich plasma: growth factors and pro- and antiinflammatory properties. Journal of periodontology 78(4): 661-669.

Fernández-Barbero JE, Galindo-Moreno P, Avila-Ortiz $\mathrm{G}$, et al. (2006) Flow cytometric and morphological characterization of platelet-rich plasma gel. Clinical oral implants research 17(6): 687-693.

Freymiller EG and Aghaloo TL (2004) Platelet-rich plasma: ready or not? Journal of oral and maxillofacial surgery: official journal of the American Association of Oral and Maxillofacial Surgeons 62(4): 484-488.

Ganapathy DM, Kannan A and Venugopalan S (2017) Effect of Coated Surfaces influencing Screw Loosening in Implants: A Systematic Review and Meta-analysis. World Journal of Dentistry 8(6): 496-502.

Ghidini G, Manfredi M, Giovannacci I, et al. (2017) Medication-related osteonecrosis of the jaw: risk factors in patients under biphosphonate versus patients under antiresorptive-antiangiogenic drugs. Minerva stomatologica 66(4). europepmc.org: 135-140.

Gupta P, Ariga P and Deogade SC (2018) Effect of Monopoly-coating Agent on the Surface Roughness of a Tissue Conditioner Subjected to Cleansing and Disinfection: A Contact Profilometric Study. Contemporary clinical dentistry 9(Suppl 1): S122S126.

Hammond JW, Hinton RY, Curl LA, et al. (2009) Use of Autologous Platelet-rich Plasma to Treat Muscle Strain Injuries. The American journal of sports medicine 37(6). SAGE Publications Inc STM: 1135-1142.
Hermeto LC, Rossi R de, Pádua SB de, et al. (2012) Comparative study between fibrin glue and platelet rich plasma in dogs skin grafts. Acta cirurgica brasileira / Sociedade Brasileira para Desenvolvimento Pesquisa em Cirurgia 27(11): 789-794.

Jain AR (2017a) Clinical and Functional Outcomes of Implant Prostheses in Fibula Free Flaps. World Journal of Dentistry 8(3): 171-176.

Jain AR (2017b) Prevalence of Partial Edentulousness and Treatment needs in Rural Population of South India. World Journal of Dentistry 8(3): 213-217.

Jakse N, Tangl S, Gilli R, et al. (2003) Influence of PRP on autogenous sinus grafts. An experimental study on sheep. Clinical oral implants research 14(5): 578-583. Kajikawa Y, Morihara T and Sakamoto H (2008) Platelet-rich plasma enhances the initial mobilization of circulation-derived cells for tendon healing. Journal of cellular. Wiley Online Library. Available at: https:// onlinelibrary.wiley.com/doi/abs/10.1002/jcp.21368.

Latifyan S, Genot MT and Klastersky J (2016) Bisphosphonate-related osteonecrosis of the jaw: a review of the potential efficacy of low-level laser therapy. Supportive care in cancer: official journal of the Multinational Association of Supportive Care in Cancer 24(9). Springer: 3687-3693.

Marx RE, Carlson ER, Eichstaedt RM, et al. (1998) Platelet-rich plasma: Growth factor enhancement for bone grafts. Oral surgery, oral medicine, oral pathology, oral radiology, and endodontics 85(6): 638-646.

Mauceri R, Panzarella V, Maniscalco L, et al. (2018) Conservative Surgical Treatment of BisphosphonateRelated Osteonecrosis of the Jaw with Er,Cr:YSGG Laser and Platelet-Rich Plasma: A Longitudinal Study. BioMed research international 2018: 3982540.

Petrungaro PS (2001) Using platelet-rich plasma to accelerate soft tissue maturation in esthetic periodontal surgery. The Compendium of continuing education in dentistry 22(9): 729-32, 734, 736 passim; quiz 746. Ranganathan H, Ganapathy DM and Jain AR (2017) Cervical and Incisal Marginal Discrepancy in Ceramic Laminate Veneering Materials: A SEM Analysis. Contemporary clinical dentistry 8(2): 272-278.

Sánchez AR, Sheridan PJ and Kupp LI (2003) Is plateletrich plasma the perfect enhancement factor? A current review. The International journal of oral \& maxillofacial implants 18(1): 93-103.

Schallmoser K, Bartmann C, Rohde E, et al. (2007) Human platelet lysate can replace fetal bovine serum for clinical-scale expansion of functional mesenchymal stromal cells. Transfusion 47(8). Wiley Online Library: 1436-1446.

Southworth TM, Naveen NB, Tauro TM, et al. (2019) The Use of Platelet-Rich Plasma in Symptomatic Knee 
Osteoarthritis. The journal of knee surgery 32(1): 37-45.

Spotnitz WD (2014) Fibrin Sealant: The Only Approved Hemostat, Sealant, and Adhesive-a Laboratory and Clinical Perspective. ISRN surgery 2014: 203943.

Varghese SS, Ramesh A and Veeraiyan DN (2019) Blended Module-Based Teaching in Biostatistics and Research Methodology: A Retrospective Study with Postgraduate Dental Students. Journal of dental education 83(4): 445-450.
Weibrich G, Kleis WK, Kunz-Kostomanolakis M, et al. (2001) Correlation of platelet concentration in plateletrich plasma to the extraction method, age, sex, and platelet count of the donor. The International journal of oral Et maxillofacial implants 16(5): 693-699.

World Journal of Dentistry (2017) Evaluation of Corrosive Behavior of Four Nickel-chromium Alloys in Artificial Saliva by Cyclic Polarization Test:An in vitro Study. 8(6): 477-482. 